\title{
Conhecimento dos riscos do parto cesáreo entre gestantes atendidas no pré-natal
}

\author{
Knowledge of the risks of cesarean delivery among pregnant women attending prenatal care \\ Conocimiento de los riesgos del parto cesáreo entre las mujeres embarazadas que asisten a \\ la atención prenatal
}

Rubenilson Caldas Valois ${ }^{1 *}$, Helanno Nazareno de Freitas Lima ${ }^{2}$, Valdeangela Carvalho Vasconcelos Paiva ${ }^{2}$, Ronaldo França de Sarges ${ }^{1}$, Amanda Gonçalves de Souza da Silva1, Tamires de Nazaré Soares ${ }^{3}$, Rubia Danielle Caldas Valois ${ }^{3}$, Marcia Helena Machado Nascimento'.

\section{RESUMO}

Objetivo: Verificar o conhecimento dos riscos associados ao parto cesáreo entre gestantes atendidas no prénatal em uma Unidade Municipal de Saúde. Métódos: Estudo do tipo descritivo com abordagem qualitativa, participaram 12 gestantes entre 18 a 38 anos atendidas em uma Unidade Básica de Saúde no município de Belém no estado do Pará, onde coletou-se as informações através de entrevista semiestruturada; a análise dos dados foi conduzida a partir da proposta de análise de Bardin; o estudo foi aprovado pelo comitê de ética em pesquisa da Faculdade Metropolitana da Amazônia. Resultados: a partir da análise dos dados emergiram as seguintes categorias: Caracterizando as participantes do estudo segundo o perfil socioeconômico; Compreensão das gestantes sobre os riscos relacionados ao parto cesáreo; e Principais informações recebidas no pré-natal sobre os riscos relacionado ao parto cesáreo; observando-se que a maior parte das gestantes desconhecem os riscos da cesareana. Conclusão: A maior parte das entrevistadas não conhecem o real risco da cesareana, e em muitas situações são induzidas a realizar esta cirurgia ou até mesmo este procedimento é imposto por parte dos profissionais de saúde. Espera-se a partir do estudo contribuições com o debate sobre o conhecimento dos riscos do parto cesáreo.

Palavras-chave: Gestante, Conhecimento, Cesárea.

\begin{abstract}
Objective: To verify the knowledge of the risks associated with cesarean delivery among pregnant women attending prenatal care at a Municipal Health Unit. Methods: This was a descriptive study with a qualitative approach. Twelve pregnant women between 18 and 38 years old attended a Basic Health Unit in the municipality of Belém in the state of Pará, where the information was collected through a semi-structured interview; the analysis of the data was carried out from the proposal of analysis of Bardin; the study was approved by the research ethics committee of the Metropolitan University of Amazonia. Results: from the analysis of the data emerged the following categories: Characterizing the study participants according to the socioeconomic profile; Understanding of pregnant women about the risks related to cesarean delivery; and Main information received during prenatal care on the risks related to cesarean delivery; observing that most pregnant women are unaware of the cesarean's risks. Conclusion: Most interviewees don't know the real risk of cesarean, and in many situations are induced to perform this surgery or even this procedure is imposed by health professionals. It is expected from the study contributions with the debate about the knowledge of the risks of cesarean delivery.
\end{abstract}

Keywords: Pregnant woman, Knowledge, Cesarean section.

\footnotetext{
1 Universidade do Estado do Pará, Belém-Pará. *E-mail: rubenilsonvalois@gmail.com

2 Centro Universitário da Amazônia (UNIFAMAZ), Belém-Pará.

3 Universidade da Amazônia (UNAMA), Belém-Pará.
} 


\section{RESUMEN}

Objetivo: Verificar el conocimiento de los riesgos asociados con el parto cesárea entre las mujeres embarazadas que asisten la atención prenatal en una Unidad de Salud Municipal. Métodos: estudio descriptivo con enfoque cualitativo. Doce mujeres embarazadas entre 18 y 38 años asistieron a una Unidad de Salud Básica en el municipio de Belém, en el estado de Pará, donde la información se recopiló através de una entrevista semiestructurada; análisis de los datos se realizó a partir de la propuesta de análisis de Bardin; estudio fue aprobado por el comité de ética de investigación de la Universidad Metropolitana de la Amazonía. Resultados: Análisis de los datos surgieron las siguientes categorías: Caracterización de los participantes del estudio según el perfil socioeconómico; Comprensión de las mujeres embarazadas sobre los riesgos relacionados con parto por cesárea; La información principal recibida durante atención prenatal sobre riesgos relacionados con el parto por cesárea; observando que la mayoría de las mujeres embarazadas no son conscientes de los riesgos de cesárea. Conclusión: Mayoría de los entrevistados no conocen el riesgo real de una cesárea, y en muchas situaciones se les induce a realizar esta cirugía o incluso este procedimiento es impuesto por profesionales de la salud. Se espera de las contribuciones del estudio con el debate sobre el conocimiento de los riesgos del parto cesárea.

Palabras clave: Embarazada, Conocimiento, Cesárea.

\section{INTRODUÇÃO}

A cesariana e o parto normal são as alternativas disponíveis para o nascimento e, dessa forma, esperase que a gestante tenha o direito de analisar os riscos e benefícios para livremente optar pela via de parto. Porém, existem aspectos relacionados à assistência ao parto que ainda precisam de discussão. O modelo de assistência ao parto no Brasil é caracterizado por excesso de intervenção, o que tem contribuído para o aumento do número de cesáreas (NASCIMENTO RRP, et al., 2015).

A cesárea tornou-se importante para a assistência obstétrica e contribuiu para a redução da morbimortalidade entre as gestantes e de seus recém-nascidos, mas o exagero de sua prática tem efeito oposto, inclusive por consumir recursos preciosos do sistema de saúde. Entretanto, a utilização indiscriminada do procedimento trouxe problemas e prejuízos à saúde das gestantes e bebês, e alguns chegam a questionar se a utilização da cirurgia cesariana não teria se tornado o modo normal de nascer na atualidade (NAKANO AR, et al., 2016; TOSTES NA e SEIDL EMF, 2016).

Ao longo do tempo, a cesárea deixou de ser um método para melhorar os resultados perinatais e tornouse um produto de consumo, de tal maneira que as taxas são menores entre as mulheres mais pobres e aumentam na proporção em que aumenta o poder aquisitivo da população (SANTIAGO DC e SOUZA WKS, 2017).

Para muitos, a cesárea é relatada como o nascimento sem dor, porém, a dor pós cesariana e a recuperação tardia são vista como pontos negativos, o medo da dor e a experiência não agradável em parto anterior favorecem a escolha da cesariana, esta é descrita como algo mais complicado: um procedimento cirúrgico que tem a reabilitação dolorosa e com dependência da mulher, podendo causar limitação para cuidar do bebê (SILVA SAM, 2016).

A prática da cesárea eletiva é considerada aquela na qual não se observa indicação clínica ou obstétrica para sua realização, esta tem aumentado de forma relevante em todo o país. A principal justificativa para a elevação desse tipo de intervenção cirúrgica é a possibilidade de programação do parto o que muitas vezes é considerado conveniente para a gestante e seu médico, porém, pouca atenção tem sido dada a um fato preocupante que é a realização das cesáreas eletivas (NOVO JLVG et al., 2017).

Em se tratando de números, o Brasil é o líder mundial de cirurgias cesarianas com uma taxa de $56 \%$ do total de partos, enquanto o índice recomendado pela Organização Mundial de Saúde (OMS) é de no máximo $15 \%$. Estima-se que no Brasil quase um milhão de mulheres são submetidas a essa cirurgia sem indicação médica e obstétrica adequada, impactando no aumento das taxas de cesáreas desnecessárias (RIBEIRO LB, 2016). 
A incidência do número de cesáreas nas regiões brasileira tem aumentado a cada ano considerando-se dados do Sistema de Informações sobre Nascidos Vivos (SINASC), em 2013, as regiões Norte apresentava taxas de $44,5 \%$, Nordeste $48,4 \%$, Centro Oeste $61,4 \%$, Sudeste $60,6 \%$ e Sul $61,7 \%$. Para a Organização Mundial da Saúde (OMS), não há motivos que justifiquem proporções de partos cesáreos superiores a $15 \%$ (OLIVEIRA ACM, et al., 2016).

A OMS divulgou um alerta sobre o excesso de cesarianas desnecessárias em países como o Brasil, o líder disparado nesse tipo de parto. De acordo com o ranking da OMS, O Brasil é o único país do globo a ter mais da metade de todos os nascimentos feitos por essa cirurgia (ZANARDO GLP, et al., 2017).

Em se tratando de indicações, as da cesariana podem ser agrupadas em maternas e fetais, assim como, em absolutas e relativas. São prováveis indicações de cesarianas: herpes ativo no momento do trabalho de parto; condilomas ou outros tumores que impeçam a progressão da apresentação fetal; distorcias que podem ser de ombro, desproporção céfalo-pélvica, apresentação pélvica; gemelaridade; infecção pelo Vírus da imunodeficiência adquirida; placenta prévia central; prolapso de cordão umbilical; sofrimento fetal agudo e descolamento prematuro de placenta (CÂMARA R, et al., 2016).

Além disso, deve ser discutido os riscos associados ao procedimento, um dos principais é o processo anestésico, esta pode desencadear hemorragia graves e consequentes necessidades de transfusões sanguíneas, infecções, danos nos órgãos localizados perto do útero (como bexiga), problemas de coagulação do sangue e o bloqueio de vasos sanguíneos nos pulmões com o líquido amniótico, que pode resultar na morte da mãe ou do feto, dor crônica na zona onde foram feitas as incisões ou sensibilidade ao toque nas zonas acima ou abaixo das incisões (DIAS JA et al., 2017).

Para que haja indicação de parto cesáreo em pacientes, torna-se fundamental que alguns preceitos sejam rigorosamente observados e seguidos. Impõem-se o consentimento informado dos riscos da cirurgia aos quais a paciente será submetida ao se tentar o parto cesáreo; a monitorização da atividade uterina, quer espontânea ou induzida, há a necessidade de que as maternidades de baixo e de alto risco tenham asseguradas equipes adequadas de assistência obstétrica de plantão 24 horas (CÂMARA R, et al., 2016).

Observa-se que quando se decide pela cesárea, no momento inicial da gestação, este fato está relacionada tanto às experiências reprodutivas anteriores, próprias e de outras mulheres da família, como ao contexto sociocultural, que é marcado pelo medo da dor do parto normal. Os valores pessoais e familiares interferem nas decisões com relação à via de parto, construídas durante o pré-natal (RISCADO LC, et al., 2016).

A escolha pela cesariana sem indicação clínica, está associado ao conhecimento errôneo das gestantes em relação ao parto vaginal, muitas vezes pela falta de informação, medo da dor, a ausência de melhores informações sobre a extensão e a natureza da dor pós-parto, representa um problema significativo uma vez que as mulheres apresentam-se despreparadas para lidar com esse desconforto (ZANARDO GLP, et al., 2017).

Também não menos importante a violência obstétrica está relacionada diretamente com questões sociais como de gênero, de raça, de classe e institucional. Pode ser identificado como violência obstétrica a imposição de intervenções danosas tal como a cesárea eletiva, esta pode acarretar danos à integridade física, psicológica e moral das mulheres nas instituições em que são atendidas, bem como o desrespeito ao seu direito de escolha da via de parto (ANDRADE BP e AGGIO CM, 2014).

Algumas formas de violência obstétrica parecem ser evidentes, como as agressões verbais realizadas por profissionais de saúde. Porém, outros procedimentos ditos de rotina não são tão facilmente reconhecidos como atos violentos, tais como o parto cesáreo e as intervenções médicas utilizadas de forma desnecessária. Essas práticas proporcionam grandes riscos as gestantes por isso vem sendo cada vez menos aceita por uma boa parte das parturientes, porém ainda não são vistas como atos violentos e menos ainda são entendidas como eventos que podem ser traumáticos (ESTUMANO VKC, et al., 2017). 
A partir do exposto teve-se como objetivo desse estudo verificar o conhecimento dos riscos associados ao parto cesáreo entre gestantes atendidas no pré-natal de uma unidade municipal de saúde no município de Belém no estado do Pará.

\section{MÉTODOS}

Tratou-se de um estudo descritivo com abordagem qualitativa, realizada com 12 gestantes, utilizou-se para determinação do número de participante a técnica da saturação teórica dos dados, desta forma, observandose durante a coleta de dados a saturação das respostas o que levou a suspensão de inclusão de novas participantes, tal como proposto no desenho do estudo.

A coleta das informações foi realizada em uma Unidade Municipal de Saúde no munícipio de Belém-Pará, onde é disponibilizado uma grande demanda de atendimento voltado para a gestante através do programa de pré-natal. A unidade conta com uma equipe multiprofissional, dentre eles o enfermeiro que realiza a consulta de enfermagem do pré-natal; a unidade disponibiliza uma excelente estrutura para promover um bom funcionamento e atendimento aos usuários, onde também são realizados outros programas de saúde preconizados pelo Ministério da Saúde.

Foram incluídas na pesquisa gestante com idade entre 18 e 38 anos, e que estavam regularmente matriculadas e participantes nas atividades do programa de pré-natal da unidade, independentemente do número de gestação, e com boa capacidade intelectual e cognitivo, o estudo foi enviado ao Comitê de Ética da Faculdade Metropolitana da Amazônia, onde obteve-se o consentimento legal para realização da pesquisa mediante os princípios éticos, de acordo com a CAEE no 86040318.1.0000.5701, e parecer n² 2.587.808.

A coleta de dados foi realizada entre os meses de abril e maio de 2018 , onde inicialmente foi realizado um convite formal para as possíveis participantes da pesquisa, informando-as sobre o tema da pesquisa e seus objetivos; já no segundo momento, as que aceitaram participar do estudo, após repassadas as informações preliminares, assinaram o Termo de Consentimento Livre e Esclarecido com o intuito de garantir o anonimato e o sigilo dos dados, após isto, foram encaminhadas para o consultório de enfermagem, onde iniciou-se o terceiro momento aplicando-se o roteiro de entrevista semiestruturada com perguntas abertas, as falas foram gravadas em áudio em aparelho gravador digital e posteriormente codificadas e arquivadas de forma sigilosa pelos pesquisadores; após isto foram transcritas e processadas.

As falas das gestantes foram codificadas durante a entrevistada semiestruturada. Os códigos foram agrupados pelas semelhanças de significados em categorias específicas. As participantes do estudo foram codificadas como Gesta 01, Gesta 02 ... Gesta 12. Os dados foram analisados de acordo com conteúdo proposto por Bardin, sendo operacionalizada em três fases: Pré-análise, exploração do material e interpretação dos resultados (BARDIN L, 2016).

Na pré-análise realizou-se a leitura geral do material, de forma geral, efetuou-se a organização do material investigado, constituiu-se como uma fase intermediária, que reuniu todo o material para tratar as informações coletadas. A exploração do material foi feita para que dentre o material coletado fosse utilizado somente os elementos específicos de acordo com o propósito do estudo, sendo agrupadas tematicamente em categorias iniciais, intermediárias e finais aos quais procurou-se compreender o sentido da fala das entrevistadas.

$\mathrm{Na}$ interpretação dos resultados captou-se os conteúdos manifestos contidos em todo material coletado na entrevista. Nesta fase foi realizado uma leitura geral do material, a leitura Interpretativa, análises exaustivas do material arrecadado e adquirido a partir dos depoimentos das participantes o que ofereceu o significado mais amplo aos resultados obtidos, estes foram descritos com a finalidade de identificar o conhecimento das gestantes a respeito dos riscos do parto cesáreo.

Em seguida, realizou-se a classificação e a categorização das falas, com sínteses coincidentes e divergentes de ideias, e sua ligação, ou não, a alguma categoria. Emergindo assim as seguintes categorias: Caracterizando as participantes do estudo segundo o perfil socioeconômico; Compreensão das gestantes sobre os riscos relacionados ao parto cesáreo; principais informações recebidas no pré-natal sobre os riscos relacionado ao parto cesáreo. 


\section{RESULTADOS}

\section{Caracterizando as participantes do estudo segundo o perfil socioeconômico}

Dentre as doze gestantes participantes, no quesito de escolaridade a maioria relatou ter cursado somente até o ensino fundamental completo e somente uma relatou ter terminado o ensino superior. Em relação as atividades remuneradas, a metade do grupo declarou estar desempregada no momento sem nenhuma remuneração e que dependiam da renda familiar e de seus companheiros, a outra metade declarou exercer atividades remuneradas.

A renda familiar mensal do grupo que declarou receber remuneração variou entre um a quatro salários mínimos. Em relação à idade, os resultados obtidos evidenciaram a prevalência de parturientes jovens, com idade que variam entre 18 a 38 anos. Quanto à quantidade de gestações, a maioria declarou ser a primeira gestação, sendo o restante entre segunda e terceira gestação. Informações evidenciadas pelas seguintes falas:

"Eu tenho 27 anos estudei só até o primário e tenho um filho que nasceu de parto normal de dois anos e já estou esperando outro que tá com cinco meses na minha barriga, eu não trabalho só meu marido mesmo, ele ganha dois salários, moro em casa de alvenaria sou desempregada" (gesta 07).

"Eu estudei até o ensino fundamental incompleto não tenho filhos ainda, moro em casa de alvenaria, a doutora disse que vou ter normal, sou desempregada só meu marido é quem trabalha, nossa renda é de um salário e meio" (gesta 12).

"Eu sou mãe de uma menina e já realizei uma cesárea, já decidi com a minha médica pela segunda cesárea porque para mim é mais cômodo pois trabalho como professora e meu horário é bem curto, prefiro programar o nascimento do meu segundo filho acho a cesárea a melhor opção pra mim" (gesta 08).

\section{Compreensão das gestantes sobre os riscos relacionados ao parto cesáreo}

De acordo com a compreensão das gestantes participantes da entrevista sobre os riscos relacionados ao parto cesariano algumas relataram que:

"Eu entendo que a cirurgia da cesárea é arriscada como é uma operação a gente pode morrer se der algo errado pode subir pra cabeça, né mesmo? (Gesta 07).

"Sim, tem riscos sim porque entendo ser uma cirurgia pois recebe anestesia ou algo parecido, eu acho que sim tem risco sim porque tem que cortar, né?” (Gesta 09).

"Compreendo mais ou menos os riscos da cesárea principalmente quando ela se opera e sai do hospital, tem que ter mais repouso não pode fazer muita coisa, diferente do parto normal, o normal não tem aquele repouso, mas não é tão complicado tão difícil quanto a cesáreo, não pode fazer nada pelo fato de ter sido cortada" (Gesta 11).

"Ah! Eu entendo que o parto cesáreo não deixa de ser uma cirurgia, o risco que a gente corre é de uma cirurgia normal a gente pode morrer no caso, a gente não sabe se tem algum problema de saúde né? Como eu posso morrer de uma parada cardíaca alguma coisa eu acho que a gente corre muito mais riscos no parto cesáreo do que no parto normal né? E sem contar com a recuperação né? Que é mais rápido no parto normal" (Gesta 01).

"Eu compreendo é que a gente tem que ficar 40 dias de repouso não posso andar muito, não pode fazer esforço, não pode fazer força, tem que ter cuidado na anestesia, na aplicação da anestesia pra não correr o risco de ficar paralitica essas coisas é que eu escuto de mãe e de vó" (Gesta 03). 
"Pode acontecer muitos riscos né! Eu acho assim que depende de que forma for, tanto cesáreo como o normal corre risco, eu acho, tudo é o momento, mas não sei dizer nenhum risco não falam disso" (Gesta 05).

\section{Principais informações recebidas no pré-natal sobre os riscos relacionado ao parto cesáreo}

Quando questionadas sobre as informações recebidas ao longo do pré-natal sobre os possíveis riscos relacionados ao parto Cesário as participantes relataram as seguintes informações:

"Ah! em nenhum momento é passado informações sobre o parto cesáreo, eu acredito que o público é sempre levado pra essa questão do parto normal, nunca é pelo parto cesáreo, só quando é de risco e não tem outra opção, nunca é passado nenhuma informação pra gente, a cesárea é uma cirurgia mas nunca é passado, assim não é um tema que é abordado com frequência no nosso dia a dia, não sei nadinha do risco" (Gesta 04).

"Não nos repassam informações sobre os riscos de parto cesáreo pouco nos falam do parto normal, só sei que os pontos podem inflamar e que a recuperação demora mais tempo, só isso que eu entendo até agora" (Gesta 06).

"O risco né! É por causa dos pontos que inflama, quando não tem repouso adequado pode até abrir né! Só isso não tenho muito conhecimento assim de parto cesáreo porque não foi falado pra mim assim de parto cesáreo" (Gesta 02).

"Tem que falar sobre a cirurgia, eles não falam nada disso! só sabem perguntar se estar tudo bem? De riscos de parto cesáreo não falam nada" (Gesta 08).

"Não sei do risco! Até porque no meu pré-natal não falam de risco e nem de cirurgia de cesárea nenhuma. A minha irmã fez essa tal de cesárea e ela era alérgica a medicamento, deu nela uma coisa ruim por causa de um remédio que deram pra ela, e quase ela morre. Mas não sei riscos nenhum não" (Gesta 10).

"Não sei dizer não de riscos dessa cesárea! Já me falaram para realizar essa cesárea porque é mais rápida, mas tô com medo de fazer porque me disseram que é uma cirurgia que corta a gente, vou até me informar melhor desses riscos" (Gesta 12).

\section{DISCUSSÃO}

Observou-se nos resultados deste estudo um grupo de mulheres jovens e que a maioria apenas tinha cursado o ensino médio, reforçando o que diz a literatura, que fatores socioeconômicos e escolaridade podem influenciar na escolha da via de parto, principalmente pela falta de autonomia e desconhecimento de seus direitos, e percepção equivocada sobre o processo parturitivo, fato este reforçado pelo autores a seguir.

A diferença racial, o estrato sócio demográfico, a renda e a escolaridade influenciam a percepção das usuárias sobre o atendimento ao parto e ao parto em si, muitas vezes são consideradas sem autonomia e sem capacidade de decidir sobre seu próprio corpo e tipo de parto (NASCIMENTO RRP, et al., 2015).

A escolha da mulher pelo parto normal ou pela cesariana está relacionada a fatores socioeconômicos, a maior parte delas optaria pelo parto normal devido à rápida recuperação, sendo esta cirurgia indicada apenas em casos em que o parto normal ofereça riscos à parturiente e seu filho. Contraditoriamente os índices de cesarianas ainda superam os do parto natural, à medida em que as mulheres aumentam seu poder aquisitivo passam a optar mais pela cirurgia cesariana, mesmo com campanhas de incentivo para a realização de parto natural (DOMINGUES RMSM, et al., 2014). Não obstante, percebeu-se entre as participantes que as mesmas não tinham a real percepção sobre os riscos de se submeter a um procedimento cirúrgico de forma desnecessária, em muitas situações associando apenas ao risco da anestesia, ou equiparando as outras cirurgias de forma genérica, não conseguindo associar aos riscos específicos da cesariana. 
A cesariana é uma cirurgia que submete a mulher gestante a um risco de morte dez vezes maior quando comparado ao parto vaginal. Os riscos inerentes a este procedimento vão desde hemorragias, infecções pósparto, embolia pulmonar, riscos com anestesia até mesmo a morte. Para os recém-nascidos, os riscos deste procedimento estão associados a possibilidade de distúrbios respiratórios, icterícias, risco de prematuridade, anóxia perinatal até a morte neonatal, além de estar relacionado com o aumento das taxas de internação em Unidades de Terapia Intensiva Neonatal (ANJOS CS, et al, 2014).

Em nenhum momento foi explicitado pelas gestantes entrevistadas, os principais motivos que indicam a realização da cesárea, nem a associação com o risco de morte em decorrência deste procedimento, em muitos casos desnecessários, podemos perceber a importância disso a partir da visão dos autores a seguir.

Dentre as indicações de cesáreas os riscos devem sempre ser avaliados uma vez que o parto vaginal pode ser possível e não está contraindicado. Apesar de aumentar o risco de morbimortalidade tanto para mãe quanto para o recém-nascido muitas gestantes desejam a cesárea como via de parto por acharem mais rápido, prático e menos doloroso, mas em muitas situações estas mulheres desconhecem as complicações que a cirurgia cesariana pode acarretar (SILVA ACL, et al., 2017).

O que se observou no estudo realizado, é que claramente a escolha pela via de parto estava atrelada, principalmente, a questão da dor, sendo influenciada pela decisão de outras pessoas, tais como familiares e profissionais de saúde.

Optar por um parto seguro, sem dor e sem riscos é a preferência de cada gestante, durante a gestação inicial muitas mulheres escolhem o parto via vaginal, mas ao aproximar o dia de parir elas passam a ter outra escolha, optando pela via cirúrgica, muitas vezes induzida pelo profissional obstetra ou pela imposição médica final, não levando em consideração sua aceitação ou não em relação à conduta a ser tomada, nem a associação entre a sua aceitação e os riscos em que estas são expostas (MATOS TS, et al., 2017).

O papel dos profissionais de saúde é preponderante na decisão da via de parto, assim como, no empoderamento da mulher e de sua família nesta escolha, mas evidenciamos que em muitas situações os profissionais que atendem esta mulher no serviço de pré-natal não as informa de forma qualificada, e em muitos momentos até reforça a escolha pela via de parto menos segura, retirando a autonomia da mulher no processo de decisão.

Para que seja possível fortalecer a autonomia da mulher na escolha do parto é imprescindível que os profissionais de saúde trabalhem em prol do empoderamento da gestante. Empoderar a gestante é fornecer a ela conhecimento e dar suporte para que sua autonomia seja fortalecida. O empoderamento da gestante amplia o seu conhecimento sobre os riscos da realização do parto cesáreo e reforça o conhecimento do seu papel na escolha de seu parto, tornando-a capaz de exercerem escolhas conscientes para si (KOTTWITZ F, et al., 2017).

A escolha pela via de nascimento pode ser influenciada por diversos fatores, tais como: os riscos e benefícios; possíveis complicações no procedimento e as possíveis repercussões no futuro. Auxiliando neste processo, as gestantes devem receber suporte através de informações qualificadas que lhes auxiliarão no processo decisório da melhor via de nascimento, respeitando-se desta forma sua decisão informada, tais informações devem ser ofertadas ao longo de todo acompanhamento gestacional e parturição (SILVA SPC, et al., 2014).

A orientação sobre a melhor forma de parir nos serviços de saúde deve ser compartilhada entre as gestantes durante o pré-natal, estas devem ter autonomia na decisão da escolha do parto, devem ser informadas quanto aos riscos da realização da cirurgia cesariana (FERREIRA JUNIOR AR, et al., 2017).

A partir da percepção das mulheres entrevistadas neste estudo, percebe-se que há a necessidade de maior participação dos profissionais de saúde no processo de reconhecimento de potenciais problemas relacionados as vias de parto, principalmente, os riscos e benefícios de cada tipo de parto, necessitando, desta forma, trabalhar a construção da autonomia destas mulheres na decisão sobre seus corpos. 
A qualidade da assistência no pré-natal, está vinculada à relação profissional-paciente na qual há diálogo com explicações sobre procedimentos, exames e orientações. A forma como o profissional aborda a gestante, com conversas, orientações e a capacidade de ouvi-la é positiva no momento da realização da consulta do pré-natal, principalmente às mães primíparas (SOARES ES et al., 2017).

Os cuidados preventivos, as orientações de promoção à saúde, é um diferencial do enfermeiro, este é diferente do médico que é treinado para identificar e tratar patologias, sendo assim, torna-se o profissional de enfermagem indispensável nas atividades normais do pré-natal, pelas características da profissão. 0 enfermeiro possui os pré-requisitos que irão possibilitar o atendimento humanizado, e o estabelecimento do vínculo entre suas pacientes irá estimular o profissional a utilizar sua sensibilidade para visualizar e orientar a gestante como um ser completo em toda a sua gestação (REIS DM e LOPES DAC, 2015).

\section{CONCLUSÃO}

A falta de esclarecimento sobre o risco da cesariana deixa as gestantes vulneráveis e propícias a serem induzidas por parte dos profissionais que lhe atendem no pré-natal, pela escolha do tipo de via de parto, já que supostamente estes profissionais detém o conhecimento; cabe ressaltar que embora haja a ideia de que as gestantes compreendam os riscos do parto cesáreo e tenham preferência pelo parto vaginal, o receio da dor e o medo do desconhecido acaba prejudicando na decisão final do tipo de parto a ser escolhido, causando um alto índice de cesáreas, tal como se observa no Brasil. É fundamental que todos envolvidos na assistência a gestante no pré-natal se posicionem e fiquem atentos no repasse dessas informações, e que não se limitem a falar somente dos benefícios do parto vaginal, mas também sobre os riscos que envolvem o parto cesáreo, para que todas as gestantes fiquem cientes dos riscos que elas e o seus bebês estarão expostos ao optarem ou deixarem ser induzidas pela escolha da cirurgia cesariana.

\section{REFERÊNCIAS}

1. ANDRADE BP, AGGIO CM. Violência obstétrica: a dor que cala. In: Anais do III Simpósio Gênero e Políticas Pública, 2014; Londrina (PR).

2. ANJOS CS, et al. Cesárea Desnecessária no Brasil: Revisão integrativa. Enfermagem Obstétrica, 2014 ; 86-94.

3. BARDIN L. Análise de conteúdo. São Paulo: Edições 70, 2016, 229 p.

4. CÂMARA R, et al. Cesariana a pedido materno. Rev. Col. Bras. Cir, 2016; 301-310.

5. DIAS, et al. Estudo da mortalidade materna na Região do Alto Jequitinhonha, Minas Gerais. Revista Médica de Minas Gerais, 2017; 1-7.

6. DOMINGUES RMSM, et al. Processo de decisão pelo tipo de parto no Brasil: da preferência inicial das mulheres à via de parto final. Cad. Saúde Pública, 2014; 101-116.

7. ESTUMANO VKC, et al. Violência obstétrica no Brasil: casos cada vez mais frequente. Revista Cientifica de Enfermagem, 2017; 83-91.

8. FERREIRA JUNIOR AR, et al. Discurso de mulheres na experiência do parto cesáreo e normal. Rev. Saúde.com, $2017 ; 855-862$.

9. KOTTWITZ F, et al. Via de parto preferida por puérperas e suas motivações. Escola Anna Nery, 2017; 1-8.

10. MATOS TS, et al. Parto vaginal: associação entre a preferência inicial da gestante e a via de parto final em uma maternidade de risco habitual. In ANAIS do International Nursing Congress, 2017. Belo Horizonte (MG) 01-03.

11. NAKANO, et al. Aperfeiçoando a Técnica e Normatizando a Prática: Uma Análise do Livro Obstetrícia, de Jorge de Rezende. Rev. História, Ciências, Saúde, 2016; 155-172.

12. NASCIMENTO RRP, et al. Escolha do tipo de parto: fatores relatados por puérperas. Rev Gaúcha Enferm, 2015; 119-126.

13. NOVO JLVG, et al. Indicações de partos cesáreos em hospitais de atendimento ao Sistema Único de Saúde: baixo e alto riscos. Rev Fac Ciênc Méd, 2017; 67-71.

14. OLIVEIRA ACM, et al. Fatores Maternos e Resultados Perinatais Adversos em Portadoras de Pré-eclâmpsia em Maceió, Alagoas. Rev Arq Bras Cardiol, 2016; 1-8.

15. REIS DM, LOPES DAC. Atuação do enfermeiro no pré-natal de baixo risco: uma revisão bibliográfica. Rev Fac Ciênc, 2015; 01-15.

16. RIBEIRO LB. Nascer em Belo Horizonte: cesarianas desnecessárias e prematuridade. Dissertação (Mestrado em Enfermagem) - Escola de Enfermagem. Universidade Federal de Minas Gerais, Belo Horizonte, $2016 ; 115$ p.

17. RISCADO LC, et al. A decisão pela via de parto no Brasil: temas e tendências na produção da saúde coletiva. Texto contexto - enferm. [online], 2016; 25(1): 2-10. 
18. SANTIAGO DC, SOUZA WKS. Violência Obstétrica: uma análise das consequências. Revista Científica da Fasete, 2017;148-164.

19. SILVA ACL, et al. Preferência pelo tipo de parto, fatores associados à expectativa e satisfação com o parto. Fapemig, 2017; 01-11.

20. SILVA SAM. O que diz a literatura sobre o conhecimento das mulheres sobre a via de parto: em busca de argumentos para orientação das mulheres. Monografia (Especialização em enfermagem obstétrica-Rede cegonha) - Escola de Enfermagem. Universidade Federal de Minas Gerais, Belo Horizonte, 2016; 33 p.

21. SILVA SPC, et al. Parto normal ou cesariana? Fatores que influenciam na escolha da gestante. Rev Enferm Ufsm, 2014; 01-09.

22. SOARES ES, et al. A informação de mulheres para escolha do processo de nascimento. Rev Enferm Ufpe (online), 2017, 11(12): 5472-5431.

23. TOSTES NA, SEIDL EMF. Expectativas de gestantes sobre o parto e suas percepções acerca da preparação para o parto. Rev. Temas em Psicologia, 2016; 681-693.

24. ZANARDO GLP, et al. Violência obstétrica no Brasil: uma revisão narrativa. Psicologia \& Sociedade. 2017; 1-11. 